\title{
Studies of open charm production and properties at $\mathrm{LHCb}$
}

\section{Daniel O’Hanlon*广}

INFN Bologna

E-mail: dohanlon@cern.ch

Copious production of open-charm mesons and baryons at the LHC, in addition to the flexible trigger scheme, and excellent momentum resolution, impact-parameter, and particle identification capabilities of the $\mathrm{LHCb}$ detector, results in some of the most precise measurements to-date on the production and properties of open-charm hadrons. In particular, measurements of the $D_{s}^{+}$ meson production asymmetry at $\sqrt{s}=7$ and $8 \mathrm{TeV}$, and a high precision measurement of the $\Omega_{c}^{0}$ baryon lifetime, are presented. These measurements provide valuable information to tune event generators, on non-perturbative and spectator quark effects in $\Omega_{c}^{0}$ baryon decays, and provide valuable input to future $\mathrm{LHCb}$ measurements.

The 39th International Conference on High Energy Physics (ICHEP2018)

4-11 July, 2018

Seoul, Korea

* Speaker.

${ }^{\dagger}$ On behalf of the LHCb collaboration. 


\section{Introduction}

Measurements of the production and properties of open-charm hadrons give a window on low-energy non-perturbative QCD, and provide essential input into theoretical calculations that improve the precision and reach of flavour-physics investigations. Due the copious production of charm hadrons at the LHC and the flexible LHCb trigger programme, LHCb is in a position to make numerous measurements of open-charm hadrons with world-leading precision.

The LHCb detector [1] is a forward spectrometer, optimised for the study of $b$ - and $c$-hadron decays, and fully instrumented in the $2<\eta<5$ pseudorapidity region. High resolution tracking close to the interaction point results in an impact parameter resolution of of $20 \mu \mathrm{m}$ for high transverse momentum tracks, and a decay-time resolution of approximately $50 \mathrm{fs}$. Efficient discrimination of charged hadrons with momenta in the range $1-100 \mathrm{GeV}$ is achieved with two ring-imaging Cherenkov detectors.

\section{Measurement of $D_{s}^{+}$meson production asymmetries at $\sqrt{s}=7$ and $8 \mathrm{TeV}$}

Production asymmetries of charm hadrons produced in $p p$ collisions are thought to be driven by the effect of the proton valence quarks in the $c \bar{c}$ hadronisation process. As such, anti-charm quarks preferentially form mesons, whereas charm quarks preferentially form baryons. This difference gives rise to kinematic differences between the charm and anti-charm mesons produced, and therefore an observable charge asymmetry within the LHCb detector acceptance. However, as the $D_{s}^{+}$meson $^{1}(c \bar{s})$ does not contain any of the proton valence quarks, such a process is thought only to contribute indirectly to the observed charge asymmetry, defined as $A_{\mathrm{P}}\left(D_{s}^{+}\right)=$ $\left[\sigma\left(D_{s}^{+}\right)-\sigma\left(D_{s}^{-}\right)\right] /\left[\sigma\left(D_{s}^{+}\right)+\sigma\left(D_{s}^{-}\right)\right]$, where $\sigma\left(D_{s}^{ \pm}\right)$is the inclusive prompt production crosssection. As such, measurements of this quantity probe non-perturbative aspects of the hadronisation process.

Here, the production asymmetry is measured [2] using promptly produced $D_{s}^{+}$mesons decaying to the $D_{s}^{+} \rightarrow \phi\left(K^{+} K^{-}\right) \pi^{+}$final state, where the number of $D_{s}^{ \pm}$mesons are estimated using a maximum-likelihood fit in bins of $D_{s}^{+}$rapidity and transverse momentum. The raw asymmetry in the observed number of $D_{s}^{+}$mesons, $A_{\text {raw }}$, is corrected for the detection asymmetry of the final-state hadrons, $A_{\mathrm{D}}$, and the asymmetry due to $D_{s}^{+}$mesons produced non-promptly from $B$ meson decays, $A_{\mathrm{P}}(B)$, with relative fraction $f_{\mathrm{bkg}}$, such that

$$
A_{\mathrm{P}}\left(D_{s}^{+}\right)=\frac{1}{1-f_{\mathrm{bkg}}}\left\{A_{\mathrm{raw}}-A_{\mathrm{D}}-f_{\mathrm{bkg}} A_{\mathrm{P}}(B)\right\}
$$

The value of $f_{\mathrm{bkg}}$ is found to be $4.12 \pm 1.23 \%$, using a combination of simulated data, and known cross-sections and branching fractions. The production asymmetries of $B$ hadrons are taken from previous LHCb measurements, and overall the quantity $f_{\mathrm{bkg}} A_{\mathrm{P}}$ is a small $\left(\mathscr{O}\left(1 \times 10^{-4}\right)\right)$ correction to the raw production asymmetry. Detection asymmetries are determined using data-driven methods, and arise from the hardware and software triggers, pion and kaon-pair tracking asymmetries, and from the PID requirements.

\footnotetext{
${ }^{1}$ Charged-conjugate decays are implied throughout, except in the definition of asymmetries.
} 

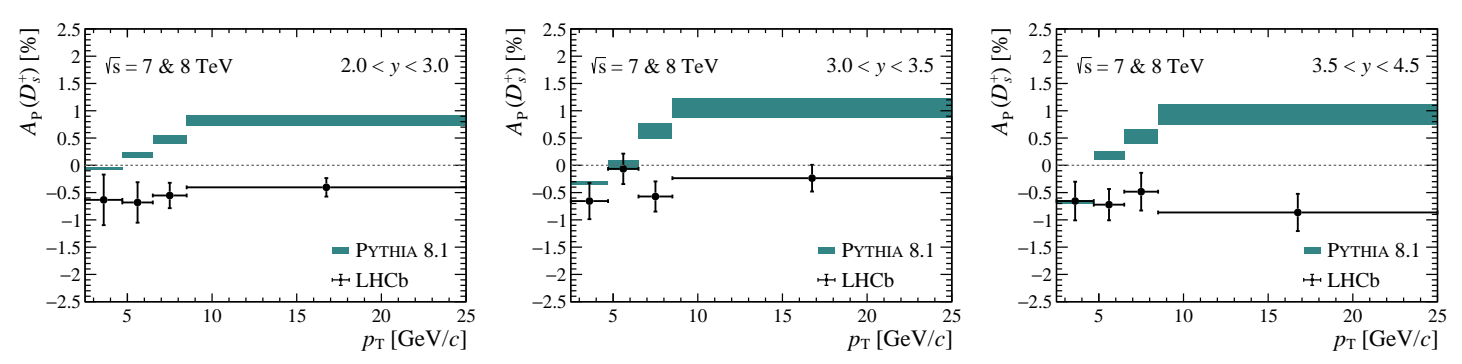

Figure 1: Production asymmetry in rapidity ranges of $2.0<y<3.0$ (left), $3.0<y<3.5$ (centre), $3.5<$ $y<4.5$ (right), for the combined 7 and $8 \mathrm{TeV}$ datasets. The LHCb measurements (points with error bars) are compared with the Pythia 8.1 predictions (green bands) in each rapidity range, in bins of transverse momentum, where the Pythia predictions account for statistical uncertainties only.

Results of the production asymmetry measurements for the combined 7 and $8 \mathrm{TeV}$ datasets can be seen in Figure 1. The predictions from Pythia 8.1 yield a moderate positive asymmetry in all rapidity regions, with a strong dependence on transverse momentum. However the LHCb results indicate a smaller, negative production asymmetry, with no pronounced dependence on transverse momentum. Overall, the LHCb results indicate a 3.3 standard-deviation difference from the hypothesis of zero production asymmetry for $D_{s}^{+}$mesons at 7 and $8 \mathrm{TeV}$, within the $\mathrm{LHCb}$ detector acceptance, corresponding to a value of $A_{\mathrm{P}}\left(D_{s}^{+}\right)=(-0.52 \pm 0.13$ (stat $) \pm 0.10$ (syst) $) \%$. These results are in agreement with, and supersede, previous $\mathrm{LHCb}$ measurements of this quantity at $7 \mathrm{TeV}$ [3].

\section{Measurement of the $\Omega_{c}^{0}$ baryon lifetime}

Measurements of heavy-hadron lifetimes are useful inputs for non-perturbative terms in the heavy quark expansion (HQE) technique used for predictions in quark-flavour physics. Charm hadrons in particular are important: the small mass of the $c$-quark compared to the $b$-quark can result in the size of these higher-order non-perturbative terms, those related to the contribution of spectator quarks in the decay, being substantial. The lifetime of charm mesons are well known, with all lifetimes being known to a relative precision of $1 \%$ or better, however the lifetime of charm baryons are comparatively less well known, with the lifetime of the $\Omega_{c}^{0}$ baryon known only to a relative precision of $17 \%$.

Here a measurement of the ratio of the $\Omega_{c}^{0}$ and $D^{+}$hadron lifetimes is performed, using $3.0 \mathrm{fb}^{-1}$ of LHC Run 1 data from the LHCb experiment [4]. The $\Omega_{c}^{0}$ baryon is reconstructed using $\Omega_{b}^{-} \rightarrow \Omega_{c}^{0} \mu^{-} \bar{v}_{\mu} X$ decays, where $\Omega_{c}^{0} \rightarrow p K^{-} K^{-} \pi^{+}$, and the $D^{+}$meson is reconstructed using $B \rightarrow D^{+} \mu^{-} \bar{v}_{\mu} X$ decays, where $D^{+} \rightarrow K^{-} \pi^{+} \pi^{+}$. In each case, $X$ denotes additional unreconstructed particles. In each case, to reduce combinatorial background and the contribution from promptly produced charm hadrons, it is required that neither the final-state hadrons nor the charm hadron are consistent with originating from a primary vertex. The decay-time distribution of the selected charm hadrons, as measured by the difference between the reconstructed beauty and charm hadron decay vertices, is extracted using the sPlot technique [5], with a binned maximum likelihood fit to the invariant mass distribution of the final-state hadrons. The subsequent yield of $\Omega_{c}^{0} \rightarrow p K^{-} K^{-} \pi^{+}$candidates is $978 \pm 60$, and the decay-time spectrum of $\Omega_{c}^{0}$ and $D^{+}$hadrons can 
be seen in Figure 2. From these distributions, the ratio of the $\Omega_{c}^{0}$ and $D^{+}$lifetimes are found to be
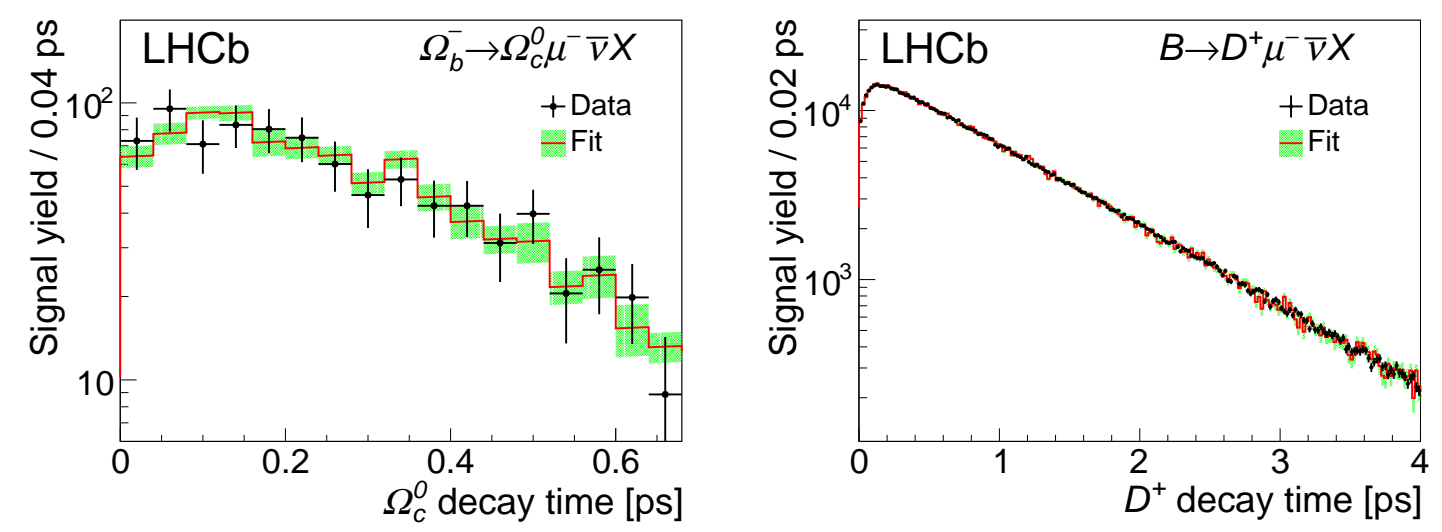

Figure 2: Decay-time distributions of $\Omega_{c}^{0}$ baryons (left), obtained from $\Omega_{b}^{-} \rightarrow \Omega_{c}^{0} \mu^{-} \bar{v}_{\mu} X$ decays, and $D^{+}$ mesons (right), obtained from $B \rightarrow D^{+} \mu^{-} \bar{v}_{\mu} X$ decays.

$$
\frac{\tau_{\Omega_{c}^{0}}}{\tau_{D^{+}}}=0.258 \pm 0.023 \pm 0.010
$$

and therefore using the world-average value of the $D^{+}$lifetime, the $\Omega_{c}^{0}$ lifetime is found to be

$$
\tau_{\Omega_{c}^{0}}=268 \pm 24 \pm 10 \pm 2 \mathrm{fs} .
$$

This value is approximately four times larger than, and inconsistent with, the current world-average value of $69 \pm 12$ fs from fixed-target experiments [6].

\section{Summary}

Measurements by the LHCb experiment of the production asymmetry of $D_{s}^{+}$mesons at $\sqrt{s}=7$ and $8 \mathrm{TeV}$, and of the lifetime of the $\Omega_{c}^{0}$ baryon, are presented. These measurements provide valuable input to non-perturbative contributions to QCD calculations, give indications on the relative importance of various competing contributions to charm baryon lifetimes, and will be used as input to future $\mathrm{LHCb}$ analyses.

\section{References}

[1] LHCb collaboration, A. A. Alves Jr. et al., The LHCb detector at the LHC, JINST 3 (2008) S08005

[2] LHCb collaboration, R. Aaij et al., Measurement of $D_{s}^{ \pm}$production asymmetry in pp collisions at $\sqrt{s}=7$ and $8 \mathrm{TeV}$, JHEP 08 (2018) 008, arXiv: 1805.09869.

[3] LHCb collaboration, R. Aaij et al., Measurement of the $D_{s}^{+}-D_{s}^{-}$production asymmetry in $7 \mathrm{TeV} \mathrm{pp}$ collisions, Phys. Lett. B713 (2012) 186, arXiv: 1205.0897.

[4] LHCb collaboration, R. Aaij et al., Measurement of the $\Omega_{c}^{0}$ baryon lifetime, Phys. Rev. Lett. 121, 092003 (2018), arXiv: 1807.02024.

[5] M. Pivk and F. R. Le Diberder, sPlot: A statistical tool to unfold data distributions, Nucl. Instrum. Meth. A555 (2005) 356, arXiv: physics $/ 0402083$.

[6] Particle Data Group, M. Tanabashi et al., Review of particle physics, Phys. Rev. D98 (2018) 030001 